\title{
¿Qué entendemos por inseguridad alimentaria? La impactante situación en Argentina
}

\author{
What do we understand by food insecurity? The shocking situation in Argentina
}

Gabriel Bulgach $^{\mathrm{a}}$

\begin{abstract}
Resumen
En esta editorial se analiza el concepto de seguridad alimentaria y se desarrollan tres de sus dimensiones (disponibilidad, acceso y utilización). Se destaca que la dimensión de disponibilidad (producción) está en una curva creciente, al tiempo que se plantea la duda sobre el impacto en la salud por los sistemas de producción actual. Esclarece acerca de la situación de inseguridad alimentaria creciente en las dimensiones de acceso (vinculadas fuertemente a las condiciones socioeconómicas) y de utilización (indicadores de epidemia de sobrepeso y obesidad). Por último, aporta información relevada para Argentina y la contradicción de que su población atraviese situaciones de falta de acceso, siendo este país uno de los mayores productores de alimentos a nivel mundial.

Abstract

In this editorial, the concept of food safety is analyzed and three of its dimensions (availability, access and use) are considered. It is emphasized that the dimension of availability (production) has an increasing curve, while raising questions about the impact on health by current production systems. It clarifies the situation of increasing food insecurity in the dimensions of access (strongly linked to socio-economic conditions), and utilization (epidemic indicators of overweight and obesity). Finally, it provides relevant information from Argentina and the contradiction of its population attracted by situations of lack of access, being one of the largest food producers countries worldwide.
\end{abstract}

Palabras clave: Hambre, Epidemiología, Alimento. Keywords: Hunger, Epidemiology, Food.

Bulgach G. ¿Qué entendemos por inseguridad alimentaria?La impactante situación en Argentina. Evid Actual Pract Ambul. 2019;22(2):e002010.

\section{Introducción}

Como cualquier ser vivo, nosotros, los Homo Sapiens, requerimos de una cuota de energía para que nuestro cuerpo pueda desarrollar sus actividades, completar las funciones biológicas requeridas y mantenerse con vida. Sin la incorporación al organismo de determinada cantidad y calidad de "cosas", nuestro cuerpo no obtiene la energía que precisa para funcionar y muere. Esta cuota energética, dada nuestra biología, se obtiene de los alimentos. No hay nada, quizás por el momento, que reemplace a los alimentos como fuente de energía para nuestro normal funcionamiento.

A lo largo de buena parte de la historia de la humanidad, una actividad central de la vida de las personas ha sido la obtención de alimentos. La lucha contra el hambre ha sido uno de los impulsos básicos que han estructurado la vida cotidiana y la organización social de los seres humanos a lo largo de nuestra historia.

En la medida que fueron avanzando la ciencia y la tecnología, y tambien la "moral" social, los seres humanos hemos transformado nuestros modos de organización y de acción. De esta manera, la ocupación del tiempo en la obtención de alimentos se ha ido reduciendo a un mínimo para la mayor parte de la población mundial conglomerada en los centros urbanos, debido a que estas personas solo deben concurrir a lugares específicos durante un lapso muy corto, para conseguir los alimentos que su biología demanda.

El nivel y la diversidad en la producción de alimentos actual permite afirmar que, con los medios adecuados, la nutrición ha salido del campo de la necesidad y ha entrado al ámbito de la cultura.

En estos tiempos, prácticamente se ha desterrado la posibilidad de grandes hambrunas como las padecidas en distintas latitudes y momentos de la historia de la humanidad. Si, por alguna circunstancia imprevista o fenómeno natural extraordinario, algún rincón del planeta se viera fuertemente afectado por la disponibilidad de alimentos, la misma organización social tiende a dar respuesta de manera veloz y eficaz para evitar la muerte por hambre.

En este sentido, sorprende el último informe de la Organización de las Naciones Unidas para la Alimentación y la Agricultura (FAO), en el que en su prólogo se afirma que ",,en las últimas ediciones del informe se mostraba que la disminución del hambre de la que el mundo se había beneficiado durante más de un decenio llegaba a su fin y que el hambre aumentaba de nuevo,,". Más adelante señala que ",otro hecho alarmante es que cerca de 2000 millones de personas padecen inseguridad alimentaria moderada o grave en el mundo. La falta de acceso regular a alimentos nutritivos y suficientes que estas personas padecen las pone en un mayor riesgo de malnutrición y mala salud..."1,2 .

\section{Seguridad alimentaria}

En la Declaración de la Cumbre Mundial sobre Seguridad Alimentaria, desarrollada en Roma en 2009, se acordó una definición que plantea que "...existe seguridad alimentaria cuando todas las personas tienen en todo momento acceso físico, social y económico a suficientes alimentos, inocuos y nutritivos, para satisfacer sus necesidades alimenticias y sus preferencias en cuanto a los alimentos, a fin de llevar una vida activa y sana..." 3 .

Esta definición plantea entonces cuatro dimensiones primordiales de la seguridad alimentaria:

La disponibilidad física de los alimentos. El acceso económico y físico a los alimentos. La utilización de los alimentos. La estabilidad en el tiempo de las tres dimensiones anteriores.

\section{La disponibilidad}

Esta dimensión de la seguridad alimentaria aborda la cuestión de la "oferta", y es función del nivel de producción de alimentos,

a Centro de Medicina Familiar y Comunitaria San Pantaleón. gbulgach@gmail.com 
los niveles de existencia y el comercio neto. Para esta dimensión, la variable de mayor impacto es la producción.

En el análisis de la disponibilidad, la $\mathrm{FAO}^{2}$ propone la construcción de las Hojas de Balance de Alimentos. Los diferentes componentes del sistema alimentario de un país (origen vegetal más animal), en forma agregada, informan o permiten calcular su disponibilidad para el consumo.

Las variables de entrada al sistema alimentario son la existencia inicial de alimentos, la producción y las importaciones (comerciales y donaciones); mientras que las variables de salida del sistema son el consumo (humano y no humano), las exportaciones y las existencias finales.

Como sostiene la FAO: "...las hojas de balance de alimentos anuales muestran las tendencias del suministro nacional de alimentos general, dan a conocer los cambios que podrían haberse producido en los tipos de alimentos consumidos y ponen de manifiesto hasta qué punto el suministro de alimentos del país es suficiente en relación con las necesidades nutricionales...".

A través de su relación con variables demográficas, se obtiene la disponibilidad per cápita (DPC) expresada en kg/persona/año.

La cantidad de factores que hacen al análisis de la disponibilidad determinan la gran complejidad de su análisis. No sólo intervienen el conjunto de variables que inciden en la producción (cantidad de tierra cultivable, régimen de lluvias, variaciones de temperaturas, procesos de desertificación, calidad de semillas, uso de agroquímicos, productividad de los suelos, antibióticos, introducción de mejoras biológicas, etc.) sino, también, condiciones específicas de las relaciones políticas y comerciales entre países, niveles de precios internacionales, aranceles, decisiones de políticas alimentarias en cada país (determinantes del destino de excedentes o sus cuotas), niveles de otros destinos que no sean el consumo humano, tolerancia sobre desperdicios, etc.

La historia de la humanidad está repleta de episodios de hambrunas que han provocado la muerte de miles de personas. Si se pensara en términos de seguridad alimentaria, la dimensión crucial para explicar las hambrunas históricas, se relaciona con esta dimensión de disponibilidad. Las catástrofes naturales (incendios, terremotos, granizadas, sequías, etc.), las plagas en los cultivos por hongos y bacterias, las guerras prolongadas, explican en buena medida las deficiencias en la disponibilidad de alimentos en la historia.

Actualmente, el nivel de mundialización, la existencia de cooperación internacional y la conciencia social, mantienen un colchón de auxilio frente a cualquier evento que incida en la disponibilidad de alimentos en algún punto del planeta.

Un interesante informe del Instituto Nacional de Tecnología Agropecuaria (INTA), en el que se analiza la evolución de la disponibilidad de alimentos entre 1963 y 2013 a nivel mundial, plantea que "...la disponibilidad del conjunto de alimentos per cápita a nivel mundial, en los últimos cincuenta años se ha incrementado un 33,3\%. Es decir, cada persona -en teoría- dispondría de un volumen de alimentos un tercio mayor al que contaba en 1963. Partiendo ese año de una disponibilidad per cápita mundial para todos los alimentos de 763,5 kilogramos/persona/año, se alcanza en 2013 una disponibilidad equiparable de 1.017,1 kilogramos/persona/año..." 4 .

\section{El acceso}

La dimensión de acceso hace referencia a la "demanda" y es función de los ingresos, del nivel de precios, así como de la existencia, densidad y cobertura de redes de protección social.

Los medios de acceso a los alimentos incluyen el comercio, el trueque, la caza, la pesca, la recolección de alimentos silvestres, la producción familiar y las redes de apoyo comunitarias.
La densidad del nivel de mercantilización en la mayor parte de los países define que la cuestión del acceso a los alimentos se produzca por la vía comercial. Dado que la mayoría de las personas en el mundo compramos nuestros alimentos, se hace evidente entonces que la dimensión de acceso tiene una fuerte correlación con los niveles de ingresos.

La dimensión de acceso se vincula fuertemente con las condiciones socioeconómicas. Estas se refieren a los salarios, la inflación, los índices de empleo, los precios de los alimentos, la concentración de los mercados y las políticas fiscales. Pero también a la composición de los hogares, la presencia de niños, el lugar y características de la residencia, la condición de la jefatura de hogar, los niveles educativos alcanzados, las condiciones sanitarias de las viviendas y las diversas variables que afectan y develan las condiciones de existencia.

La dimensión de acceso puede mejorarse positivamente con diversos mecanismos de redistribución de los ingresos. En este sentido, políticas sociales específicas tienen impacto en la dimensión de acceso, ya sea por la entrega directa de alimentos, por el establecimiento de espacios a donde las personas van a comer (comedores, merenderos, refuerzos alimentarios en las escuelas), o por la vía de programas de asignación monetaria. Subsidios específicos (fiscales, a servicios, etc.) también podrían tener fuerte impacto en la dimensión de acceso, en la medida que mejoren la proporción de ingresos de individuos y hogares que se destinan para la compra de alimentos.

La dimensión de acceso se mide a partir de entrevistas directas a la población en la que se consigna la prevalencia de la inseguridad alimentaria por falta de recursos económicos o de otro tipo. De este modo, hay tres categorías en las que cualquier persona podría clasificarse: en situación de seguridad alimentaria o inseguridad alimentaria leve, inseguridad alimentaria moderada 0 inseguridad alimentaria grave.

De acuerdo a la FAO “...las personas que experimentan una inseguridad alimentaria moderada afrontan incertidumbres sobre su capacidad para obtener alimentos y se han visto obligadas a reducir, en ocasiones durante el año, la calidad o la cantidad de alimentos que consumen debido a la falta de dinero u otros recursos. Esta hace referencia, por tanto, a una falta de acceso continuado a los alimentos, lo cual disminuye la calidad de la dieta, altera los hábitos alimentarios normales y puede tener consecuencias negativas para la nutrición, la salud y el bienestar. En cambio, en el caso de las personas que afrontan una inseguridad alimentaria grave es probable que se hayan quedado sin alimentos, hayan experimentado hambre y, en las situaciones más extremas, hayan pasado varios días sin comer, lo cual pone su salud y bienestar en grave riesgo" 1 .

En este último estudio se consigna que el 17,2\% de la población (1300 millones de personas) han atravesado una situación de inseguridad alimentaria moderada en el último año, pero "...si se combinan los niveles moderado y grave de inseguridad alimentaria, la estimación asciende al $26,4 \%$ de la población mundial o a un total de alrededor de 2000 millones de personas" 1 .

\section{La utilización}

Esta dimensión incluye aspectos que hacen a la utilización biológica de los alimentos para lograr un estado de bienestar nutricional en el que se satisfagan las necesidades fisiológicas. En este sentido, se asocian a los indicadores de nutrición.

La higiene y el saneamiento, la calidad del agua, las prácticas de cuidado de la salud, y la calidad e inocuidad de los alimentos son todos elementos que determinan el buen aprovechamiento de los alimentos por parte del cuerpo.

Las innovaciones en biotecnología en todo el sistema de producción de alimentos, la contaminación de suelos y aguas por 
fumigación y desechos industriales, entre otros factores, ponen un gran manto de duda acerca de la calidad y la inocuidad de los alimentos disponibles.

Asimismo, es importante resaltar que, actualmente, se habla de una epidemia de sobrepeso y obesidad, como fuertes indicadores de malnutrición. Se estima que el sobrepeso está en aumento en todos los grupos de edades, pero especialmente entre los niños de edad escolar y adultos en todo el mundo. En particular, los niños en edad escolar disminuyen el consumo de frutas y hortalizas, elevan el consumo regular de alimentos "chatarra", gaseosas azucaradas o edulcoradas y disminuyen la práctica de actividad física. Es la propagación de este estilo de vida y las prácticas de moda, lo que afecta fuertemente el estado nutricional general.

\section{La situación mundial}

Destaquemos algunos datos consignados en el informe de FAO acerca de indicadores ligados a la nutrición:

Uno de cada siete nacidos vivos ha tenido bajo peso al nacer (20,5 millones de bebés). Esto tiene fuerte impacto en la muerte antes del mes de vida, retrasos del crecimiento, bajo cociente intelectual y mayor riesgo de sufrir enfermedades crónicas en la adultez. La prevalencia de retraso de crecimiento en niños menores de 5 años está en disminución, aunque afectó al 21,9\% en 2018 (148,9 millones de niños). El 7,3\% de los niños menores de cinco años sufre de emaciación (49,5 millones de niños). La prevalencia de sobrepeso en niños menores de cinco años ha aumentado del $5,5 \%$ en 2012 al 5,9\% en 2018. En 2016, el $32,8 \%$ de las mujeres en edad fértil padecía de anemia, La obesidad en adultos continúa aumentando y ha pasado del $11,7 \%$ en 2012 al $13,2 \%$ en 2016.

A este respecto, desde FAO sostienen que "...se requieren enfoques multifacéticos y multisectoriales para detener e invertir la epidemia de obesidad. Se necesitan políticas destinadas a proteger, promover y apoyar la lactancia materna y para aumentar la disponibilidad y asequibilidad de los alimentos nutritivos que constituyen una dieta saludable, junto con medidas para crear entornos alimentarios más saludables y limitar el consumo de grasas perjudiciales, sal y azúcares..."

\section{Seguridad alimentaria en Argentina}

Desde el siglo XIX, Argentina es uno de los mayores productores de alimentos del mundo.

Dada su extensión, sus características topográficas, geográficas y climáticas, Argentina ha desarrollado una producción agro- pecuaria de alta intensidad que, según el dicho popular, tiene la capacidad de alimentar a "400 millones de personas". Capacidad sobrada entonces para que sus aproximadamente 45 millones de habitantes estén bien alimentados.

De acuerdo al informe del INTA ${ }^{4}$, la disponibilidad interna del conjunto de alimentos per cápita (DIPC) en Argentina creció un $78 \%$ en los últimos cincuenta años (1963-2013). Partiendo de una DIPC de $1.127 \mathrm{~kg} /$ persona/año para todos los alimentos considerados y alcanzando una de $2.003 \mathrm{~kg} /$ persona/año respectivamente al final del período.

A pesar de esto, en la reciente publicación de FAO, se consigna que 14,2 millones de argentinos, un $32,1 \%$ de la población, atraviesan una situación de inseguridad alimentaria moderada 0 grave de los cuales 5 millones atraviesan una situación de inseguridad alimentaria grave.

La problemática de la seguridad alimentaria en Argentina, se centra evidentemente en las dimensiones de acceso y de utilización.

No parece haber líneas de investigación desarrolladas en nuestro país que den cuenta de la situación de inseguridad alimentaria. A partir de la realización de una búsqueda bibliográfica, se halló un estudio en la ciudad de Santa Fe publicado en 2011, donde se determinó que $31,5 \%$ de los hogares santafesinos presentaba inseguridad alimentaria, de los cuales $21,7 \%$ era leve, $7,4 \%$ moderada y $2,4 \%$ severa $^{5}$. En otro estudio desarrollado por el Observatorio de la Deuda Social ${ }^{6}$, se señaló que $11,2 \%$ del total de hogares con residencia en los principales centros urbanos de país experimentaba algún grado de inseguridad alimentaria. De este total, $6,6 \%$ de los hogares presentaba inseguridad alimentaria moderada y $4,6 \%$ severa.

$\mathrm{Si}$, como se menciona previamente, la dimensión de acceso guarda fuerte correlación con variables macro socioeconómicas como el salario, el nivel de actividad, el nivel de precios, el índice de desempleo y otros que afectan directamente el nivel de ingresos, quizás no debería sorprendernos el dato de $32,1 \%$ de prevalencia de inseguridad alimentaria relevado por FAO en 2018.

En todo caso, debe encender las alarmas, no sólo para conocer en mayor detalle la situación de inseguridad alimentaria en nuestro país, sino para que rápidamente podamos iniciar, ampliar o corregir las políticas públicas que permitan avanzar hacia los objetivos de la erradicación del hambre en la Argentina.

\section{Referencias}

1. FAO, et al.. El estado de la seguridad alimentaria y la nutrición en el mundo 2019. Protegerse frente a la desaceleración y el debilitamiento de la economía; 2019. Available from: http://www.fao.org/3/ca5162es/ca5162es.pdf.

2. FAO. Métodos para la estimación de índices comparables de prevalencia de la inseguridad alimentaria experimentada por adultos en todo el mundo. ; 2016.

3. FAO. Declaración de la Cumbre Mundial Sobre la Seguridad Alimentaria, Cumbre Mundial sobre la Seguridad Alimentaria. Roma; 2009.

4. Diaz DN, Goldberg AS, Fernandez R. Dimensiones de la Seguridad Alimentaria en el nuevo escenario global: ¿el mito del plato vacío? Evolución de la disponibilidad de alimentos per cápita en Argentina y en el mundo entre 1963. Buenos Aires: CICPES; 2013.

5. Rosso MA, Wicky MI, Nessier MC, Meyer R. Inseguridad alimentaria en la ciudad de Santa Fe: percepción de los ciudadanos . Salud Colectiva 2015;11:234-279.

6. ODSA. La inseguridad alimentaria en la Argentina. Hogares Urbanos. Argentina; 2011 